\title{
Enhanced Laparoscopic Vision improves detection of intraoperative adverse
}

events during laparoscopy

Bertrand Trillinga, , Sinara Vijayan ${ }^{\mathrm{a}}$, Cecile Goupil ${ }^{\mathrm{a}}$, Elio Kedisseha ${ }^{\mathrm{a}}$, Antoine Letouzey ${ }^{\mathrm{a}}$, Pierre Alain Barraud $^{\mathrm{a}}$, Jean Luc Faucheron ${ }^{\mathrm{a}, \mathrm{b}}$, Gaëlle Fiard ${ }^{\mathrm{a}, \mathrm{c}}$, Sandrine Voros ${ }^{\mathrm{a}, \mathrm{d}^{*}}$

${ }^{a}$ Université Grenoble Alpes, CNRS UMR 5525, Grenoble INP, CHU Grenoble Alpes, TIMC-IMAG, 38000 Grenoble, France

${ }^{b}$ Service de Chirurgie Digestive, CHU Grenoble Alpes, 38000 Grenoble, France

${ }^{c}$ Service d’Urologie, CHU Grenoble Alpes, 38000 Grenoble, France

${ }^{d}$ INSERM

*Corresponding author:

Dr Sandrine Voros

Laboratoire TIMC-IMAG

Pavillon Taillefer

Faculté de Médecine

38700 La Tronche

Sandrine.Voros@univ-grenoble-alpes.fr

Orcid Number: https://orcid.org/0000-0002-7418-2367

Original article

Word count: 4014

Abstract: 198

Figures: 4

Tables: 2 


\section{$\underline{\text { Abstract }}$}

Background: The absence of visibility of the entire surgical scene in laparoscopic surgery can lead to unforeseen intraoperative complications. An Enhanced Laparoscopy Vision System (ELViS) was developed to eliminate the blind spots of the traditional endoscope by providing a broad view of the surgical scene from a distance, thanks to two additional images. This study assessed whether the broad view provided by the Enhanced Laparoscopic Vision (ELV) helped the surgeon to detect and react to an unexpected intraoperative adverse event (simulated hemorrhage) occurring while performing a standard task.

Methods: While participants were performing a standard task (sorting pins) on a dry lab laparoscopic simulator with or without ELV, a simulated bleeding (LED lighting) was randomly triggered. Perprocedure metrics were recorded and surgeons' feedback gathered at the end of the session.

Results: Thirteen Senior surgeons participated. Mean response time was significantly reduced when using ELV, with a similar number of simulated bleeding events between both modalities. All surgeons agreed that ELV could be helpful and constitutes an acceptable increase in cognitive load.

Conclusion: In a dry lab setup, compared to traditional endoscopy, the broader field of view provided by ELV improved outcomes when dealing with unforeseen complications like bleeding.

Keywords: Enhanced Vision in Laparoscopy - Modified Laparoscopic port - Unexpected intraoperative event detection - Laparoscopy dry lab

Funding: This work was funded in part by the French National Research Agency (Agence Nationale de la Recherche) as part of the DEPORRA2 project under grant ANR-14-CE17-0009-01 and the Investments for the Future (Investissements d'Avenir) program (Labex CAMI) under grant ANR-11LABX-0004. Other costs were covered by recurrent institutional funding (CHU Grenoble, INSERM). 


\section{Highlights}

- ELViS: an enhanced vision system developed for use during laparoscopic surgery.

- Assessment of the potential added-value of an enhanced vision for laparoscopy.

- Dry lab test bench mimics a bleeding event in a blind spot for standard laparoscopy.

- $\quad$ The system improved response times for experienced surgeons.

- Surgeons’ feedback indicated a minimal increase in cognitive load.

- Surgeons declared they would use ELViS in clinical setting when available. 


\section{Introduction}

Minimally invasive surgery using laparoscopy represented a breakthrough in abdominal surgery at the end of the $20^{\text {th }}$ century, and is now considered the gold standard for many urological, digestive, and gynecologic interventions. Compared to standard laparotomy, laparoscopy improves recovery time and cosmesis, while reducing pain, overall complications, and length of hospital stay[1-3], without altering the quality of the surgery[4]. Laparoscopy can be used to treat many diseases. However, during the intervention, the surgeon has limited access and a restricted view of the surgical scene. Indeed, the endoscope inserted through the small incision magnifies the details of the dissection to allow more precise dissection of the organ of interest, but visibility of the surrounding abdominal cavity is lost. This loss of vision can be problematic, especially as surgical instruments can inadvertently encounter other structures. Blind spots can thus result in intraoperative complications such as bowel perforation[5] or hemorrhage[6,7] that may have severe consequences if not dealt with during the procedure. In addition, the lack of visibility of the surroundings may lead to the surgeon and their assistant losing the orientation of the endoscope with respect to the surgical horizon. In this event, the surgeon's cognitive workload is increased [8] as is the risk of adverse events which may affect post-operative outcome[9].

Various approaches have been explored to improve the surgeon's awareness of the surroundings of the surgical scene, including panomorph lenses[10], optical prisms[11], enhanced multiple lenses[12], and panoramic views[13]. Alternatively, some groups have proposed increasing the field of view using distributed vision systems (either with multiple individual cameras or two to three stereoscopy systems)[14-18].

In our laboratory, based on preliminary work by Tamadazte et al. [14,19], we developed an Enhanced Laparoscopy Vision System (ELViS) to overcome the blind spots inherent to laparoscopic surgery. The system is a camera trocar that has been "augmented" by two miniature cameras. After insertion of the ELViS into the abdominal cavity, the two miniaturized cameras are deployed on either side of the trocar, and a standard endoscope is inserted inside its hollow shaft (Figure 1). Since the additional cameras are placed on the abdominal wall, they provide a perspective from a distance, while the surgery is performed mainly using the traditional endoscopic view zoomed on the organ of interest. With the two additional 
side cameras, the ELViS continuously provides a full view of the surgical scene, like in laparotomy, to help eliminate the blind spots inherent to laparoscopy.

In [19], we focused on presenting the ELViS' design and a preliminary validation of its usability in a dry lab setting. With this study, our objective was to focus on another validation aspect by exploring the potential clinical added-value of the system, based on pre-clinical evaluations. We hypothesized that the Enhanced Laparoscopic Vision (ELV) provided by the two additional cameras in the ELViS could improve the surgeons' awareness of the surgical scene and how they manage adverse intraoperative events such as bleeding. The aim of the study presented here was to assess whether providing an ELV of the laparoscopic scene could improve the capacity of experienced surgeons to detect and deal with unexpected intraoperative lateral stimuli in a specifically-designed dry lab experiment.

\section{Material and Methods}

\section{$2.1 \quad$ Study setting}

\subsubsection{Abdominal training test bench}

We searched the surgical literature for a test bench that would meet our needs for the tests to be performed. Unfortunately, no appropriate test bench was found, as standard laparoscopic trainers allow the surgeon to learn to perform tasks with laparoscopic instruments. These trainers are designed to be easy to transport or store (cubic shape) but in no case represent the dimensions of an inflated abdomen in real surgeries. Wet benches simulating hemorrhage were an alternative that we considered, but the available models [20,21] did not seem easy to implement.

Faced with this lack of appropriate options, we decided to build our own testbench (Figure 2) which consisted of a curved opaque plexiglass sheet $(55 \mathrm{~cm} * 30 \mathrm{~cm})$ attached to a metal structure $(50 \mathrm{~cm} * 30 \mathrm{~cm})$ and provided a trocar entry-point. The curve in the plexiglass sheet simulated the inflated abdomen, and the surgical scene length corresponded to that of an inflated human abdomen $(\sim 25 \mathrm{~cm})$. A 10-mm trocar for the endoscope and two 5-mm trocars for insertion of instruments were inserted through small holes in the plexiglass sheet. The exercise board described below was placed on the test bench, under the plexiglass sheet, and the video feed from the three cameras (left camera, endoscope, 
and right camera) were displayed on three screens. All three screens were placed at the same height. The setup was the same for each participant.

A simplified version of the ELViS, where the system was maintained in a deployed position, was used to ensure an identical setup for all experiments. A fixed-position plastic casing was designed with the same dimensions as that of the camera "head" of the ELViS (Figure 2). Two mini-CMOS Raspberry Pi “Spy” cameras (https://www.adafruit.com/product/1937, Vertical angle: 62.2 , Horizontal angle: $48.8^{\circ}$ and resolution $3280 \times 2464$ pixels) were inserted through this casing, they had the same viewing angle and spacing as the ELViS. The casing was designed to ensure that the cameras were positioned with the same orientation and center-to-center distance as in the actual ELViS. Each camera was controlled by a Raspberry Pi mini-computer for real-time video-stream acquisition. A third Raspberry Pi camera was fixed to the proximal tip of a tube inserted through the endoscope trocar, to provide the endoscopic feed. The Raspberry Pi is a small, low-cost computer that allowed the easy, real-time recording of the three video feeds (endoscope, lateral cameras providing the ELV) for the experiments. The system was then fixed by screws to the endoscope trocar close to the plexiglass sheet: the endoscope could be inserted through the trocar as usual and move independently, while the ELV cameras' orientation was maintained similar to that of the endoscope since it was rigidly fixed to the trocar. The ELV system clearly provided a wider view of the surgical scene, especially when the endoscope was zoomed in (Figure 3).

\subsubsection{Exercise board}

The exercise was designed to study how surgeons responded to an unexpected adverse event, without impacting the time to complete a complex task or the patients' safety. During the exercise, a luminous stimulus was triggered on the side of the surgical scene, to simulate an unexpected event, such as bleeding, in a blind spot for standard laparoscopic vision. The luminous stimulus migrated progressively to the center of the field, eventually becoming visible in the periphery of the working field (endoscope view). The surgeons were asked to react to this ("bleeding”) event while continuing to perform a standard sorting task. They were asked to give equal priority to performing the task and controlling the bleeding and. The test bench was fully presented before starting the experiment. 
The surgical scene in the simulator was composed of three plates located in the center, with two breadboards to the left and right of the plates (Figure 4). The primary task was to sort 16 multi-colored pins (4 pins $\mathrm{x} 4$ colors) placed in the central plate into the two lateral plates, with two colors for each plate (4 pins x 2 colors). The breadboards had five low-intensity red LEDs each which simulated bleeding by lighting up. The surgeon could extinguish the LEDs by grasping a metallic plate placed between the two breadboards at the extremity of the board. The first sequence of LED illumination was triggered randomly between 2 and $10 \mathrm{~s}$ after the surgeon started to perform the task. Each of the 5 LEDs on one side would light successively from the side to the center of the surgical scene and remained lit until the surgeon extinguished the LED ribbon by grasping the metal plate. This luminous stimulus aimed to simulate a bleeding coming from outside the surgical scene and progressively gaining the center. Once the "bleeding” was stopped, the waiting time was randomized ( $<20$ s) before the next round of stimulus was triggered. The bleeding simulation and data/video recording was programmed in Python (https://www.python.org/) on one of the three Raspberry Pis used for video capture. Figure 4 illustrates the setup for the test. A video showing the feeds from the three cameras on the display screens is provided as supplementary material.

\subsection{Population and Experimental protocol}

A cohort of experienced laparoscopic surgeons (Digestive surgeons, Urologists and Gynecologists) working at Grenoble Alpes University Hospital were recruited for the study. All surgeons were senior surgeons familiar with laparoscopic techniques (>100 laparoscopic procedures as operating surgeon).

Each experimental session included only one participant and always started with a presentation of the ELViS project and the manipulation of the ELViS prototype under development, so that the participant would better understand its potential added-value when performing live surgery. The experimental setup and the exercise board were thoroughly described, emphasizing how the visual information from each camera was displayed. The task to be performed and the means to stop the LED signal during the exercise was explained to participants. For all exercises performed during the session, the same senior laparoscopic resident (CG) held the endoscope and provided an image centered on the surgical instruments. To allow participants to familiarize themselves with the three-screen display and the use of 
ELV, a peg-transfer exercise was performed with the three images displayed, and repeated (at least three times) until the participant felt comfortable with the setup.

Participants were randomized to start the exercise with or without ELV. In the "without ELV” setting, the side screens were turned off so that only the video feed from the endoscope was displayed. After completing the first test, the exercise was performed in the second condition (with ELV if the first round was with a standard endoscope or vice versa). Total time to complete the exercise and average time for the surgeons to respond to bleeding (LED stimulus) in the exercise were recorded throughout the experimental sessions. The average observable motion of the endoscope in the video feed was also measured using Optical flow (Gunner Farneback’s state-of-the-art algorithm[22], from the OpenCV computer vision library[23]). We used the endoscopic view to determine the average optical flow over the whole duration of the exercise. Although this value is approximate due to the incompletely static nature of the scene (the surgeon's instruments are moving), the average amplitude of displacement of the endoscope, when the endoscope was used alone, or when using the ELV. The data was analyzed to determine whether ELV altered how the assistant handled the endoscope in any way.

A questionnaire was administered at the end of the experiment (Appendix) to assess subjective parameters such as how well the experiment reflected real-life surgery and to record surgeons' feedback and impressions of ELV and the ELViS. Three display arrangements were described orally to the participants: a three-screen horizontal interface like in the experimental setup, a two-screen horizontal interface (one for the endoscope view and one with the views from the two lateral cameras), and a twoscreen vertical interface (one for the endoscope view and one with the views from the two lateral cameras). The idea of the panoramic view was described but not presented. Participants were asked which screen interface they thought would be most suitable, and if they had any suggestions as to how to improve the display setting.

\subsection{Compliance with ethical standards}

All participants signed a consent form for participation in the study and a confidentiality agreement. No ethical approval was necessary according to current French legislation, since the study did not involve interventions on live animals or humans. The authors have declared any potential conflicts of interest. 


\subsection{Data analysis}

Data is presented as mean and standard deviation. As the data did not follow a normal distribution, Wilcoxon's tests were used to compare the average response time to bleeding, the total time taken to complete the task, the level of movement of the endoscope with or without the ELViS, and the mean number of times the LED stimulus was controlled by the surgeon. Statistical analyses were performed using IBM SPSS Statistics v26 (IBM Corp., USA).

Raw data are available upon request to the corresponding authors.

\section{Results}

\subsection{Participants}

Thirteen surgeons participated in the experiment and were assisted by the same senior resident. They had a mean post-residency experience of 7.7 years (1-30). All but two participants were right-handed. Eight of the participants were senior consultants (Professor of Surgery or Attending), and the other five were Surgical Fellows with at least 1 year of practice (1-4 years). The areas of expertise of the surgeons were as follows: Digestive surgery $(n=8)$, Gynecology $(n=3)$, and Urology $(n=2)$. On average, participants performed 4.4 (3-6) laparoscopic surgeries per week.

\subsection{Bench metrics}

Table 1 summarizes the results relating to the time required to complete the task, the time before the surgeon responded to a bleeding event, the movement of the endoscope by the assistant (average optical flow throughout the exercise), and the number of times the surgeon stopped the simulated bleeding.

The mean time to respond to bleeding was significantly lower using ELV compared to conventional endoscopy (6.41 s vs $11.36 \mathrm{~s}, \mathrm{p}: 0.007)$. The number of simulated bleeding events dealt with during the exercise was similar in both modalities. All bleeding events were dealt with by surgeons. Overall, the time to complete the task was not statistically different whether ELV was used or not (138 s vs $128 \mathrm{~s}$, p:022). Two participants took more time to complete the task with ELV than with the endoscope alone, but the minimum time to complete the task was measured for a participant using ELV (78 s). There was no difference in mean normalized camera movement with or without ELV, thus the distance covered by 
the camera was similar: The person in charge of displacing the laparoscope (CG) did not seem to be influenced in her management by the presence or absence of ELV.

\subsection{Questionnaire}

Answers from the questionnaire are summarized in Table 2. Based on these answers, 76\% (10/13) of participants fully agreed that the ELV setting did not interfere with the surgical procedure. All participants agreed that the exercise designed for the purpose of this study had constraints (display, bleeding management) similar to real-life surgery (13/13). All surgeons declared they would accept to use the ELViS in clinical practice if it took less than 10 min to set up, but two declared they would only use the ELViS for long and difficult surgeries. The benefits of ELV indicated by the participants were: elimination of blind spots (instrument insertion, blind instruments manipulated by surgical assistant), early detection of adverse events, overall safety improvement, help with endoscope orientation for the assistant, assistance with display difficulties (such as grasping failure or bowel displacement), and other complex tasks. The main concerns expressed in relation to using the ELViS were price, duration of setup, learning curve for display use, and size of the equipment that had to be added to the existing laparoscopic screen. The screen setup with three horizontal screens was the preferred display configuration (10/13 participants). A small side screen located to the right of the laparoscopic image displaying the two images from the lateral cameras was also considered a good alternative by 2 participants. One participant preferred the panoramic view.

\section{Discussion}

This study aimed to determine whether providing additional vision (ELV) to the surgeon during laparoscopic surgery allowed them to detect unexpected events on a bench test simulating a bleeding event. The results, based on reaction times following onset of the simulated bleeding, indicate that detection was improved without affecting the time required to complete a complex task. Surgeons' feedback through a questionnaire indicated that the device was associated with an acceptable cognitive load. 
One of the main issues during laparoscopic surgery is the existence of "blind spots". At times, the surgeon loses sight of their instruments, and the instruments inserted and controlled by the surgical assistant. Inexperienced assistants can easily damage bowel tissue or tear organs as they attempt to orient their instruments appropriately. Undetected bowel injury may occur in $0.01-0.08 \%$ of laparoscopic procedures, and can have dramatic effects on the post-operative course[24-26]. ELV improved the mean time to stop simulated bleeding, and most participants indicated that they would use the device in the operating room.

Our laparoscopic simulator of unexpected adverse events was designed specifically for this study. To our knowledge, no existing simulator of adverse events during laparoscopic interventions has been described previously, except for wet lab simulators. These simulators consisted of a mock organ perfused with liquid that leaks in the event of injury, and are used to train for hemorrhage control [20,21]. In this case, the hemorrhage occurs on the organ on which the operator is working. One of the purposes of ELV was to be able to detect an adverse event (such as bleeding) early, even when it occurs in a blind spot for traditional laparoscopy. The wet simulators described in the literature were not appropriate to assess this aspect of adverse laparoscopic events. In addition, they represent a cumbersome experimental setup. For the purposes of our research, we developed a new dry lab laparoscopic simulator to represent an adverse event, in this case a hemorrhage starting laterally and moving progressively into the surgical area.

The study presented here has several limitations. Our laparoscopic simulator was designed specifically for this study to assess unexpected events occurring in blind spots during laparoscopic surgery and was not validated before use. Nevertheless, the participants declared that the constraints during the exercise were similar to those encountered in real surgery. In addition, our results might be underpowered because of the size of the study population (13 expert surgeons). Most of the expert surgeons in the institution agreed to participate in the study, but only 13 surgeons managed to fit the experiment into their schedule during the study period. Finally, the duration of the intervention to be performed was much shorter than a real-life surgical intervention. The full session lasted approximately 1 hour from 
the presentation of the ELViS project, to the actual experiment and gathering responses to the questionnaire.

Other groups have also attempted to improve vision for surgeons performing laparoscopic surgery. For example, Naya et al.[13] studied how a broad field of view helped novice surgeons performing nephrectomy by retroperitoneoscopy. They obtained a panoramic view by interlinking subsequent video frames acquired from the endoscope feed and displayed the image on an auxiliary screen. While the surgery was performed, the surgical scene changed with the dissection and mobilization of anatomical structures. The surgeon could then obtain a new panoramic view by starting a new iteration. Resident surgeons using the panoramic view performed the whole procedure ( $212 \mathrm{~min}$ vs $254 \mathrm{~min}, \mathrm{p}=0.0489$ ) and ligation of renal artery (69 min vs $106 \mathrm{~min}, \mathrm{p}=0.0016)$ faster and with less blood loss ( $87 \mathrm{~mL}$ vs $334 \mathrm{~mL}, \mathrm{p}=0.0005)$. However, the time from ligation of the renal artery to cutting of the renal vein, and the time after cutting the renal vein were not significantly different. ELV provides a real-time image of the surgical surroundings, which is not the case for the system developed by Naya et al., where the panoramic view must be refreshed at the surgeon's request. However, because the exercises in our study were much shorter than the average duration of real surgery (a few minutes compared to one or more hours), ELV had no impact on speed of surgery. Longer experiments would be needed to assess this aspect, although we expect that ELV could help reduce the duration of surgery for long surgeries involving complex tasks.

Other devices have also been developed to enhance laparoscopic vision. Roulet et al.[10] proposed the use of a panomorph lens at the tip of the endoscope to provide a high definition $180^{\circ}$ wide-angle view. In addition to problems with lens manufacturing, the screen display from this type of lens may seem unfamiliar as the panomorph view includes distortion. In the display setting proposed, the surgeon could attenuate distortion in the region of interest without moving the endoscope. Kobayashi et al.[11] developed a double prism automated endoscopic system that could provide a wider image by shifting the focus to the edge of the standard endoscope. However, the dimensions of the system were incompatible with insertion through a standard laparoscopic trocar. 
During our ELViS project, two Distributed Laparoscopy devices [16,27] were reported and tested successfully on animals and humans. Duration of procedure was not compared to a control group. Both systems are attached to the abdominal wall, providing a different point of view to that of a traditional endoscope. To avoid the cognitive load required to combine images from very different points of view, we chose to design an "augmented trocar" with miniature cameras to provide a similar axis of vision to that of the endoscope for the overall view of the surgical scene.

Kim et al.[18] presented a Trocar Camera Assembly which concept of use was similar to our ELViS prototype, with deployment of additional cameras after insertion of an enhanced trocar. Their prototype deployed four small cameras, and images were displayed on a screen and merged with the endoscope image. The field of view using the Trocar Camera Assembly was increased by $133 \%$ compared to a standard 10-mm endoscope. Modifications will be needed before it can be used on animals or humans as the foldable camera holders suffer from the same fragility as Tamadazte's early prototype[19]. The authors chose to merge the different images in a single display using stitching techniques. However, laparoscopic images are not optimally-adapted for stitching: in a real clinical setup, parallax issues would be encountered, resulting in phantom instruments and reconstruction inconsistencies. Thus, these types of display would be difficult to use in a clinical setting. In our system, we overcame this issue by choosing to have as few overlaps as possible between the lateral images and the endoscope.

Display of additional information during surgery might be challenging since the cognitive burden during surgery is already high. Adding two screens to the surgeon's view could become cumbersome. Understanding the views provided by the ELViS on the lateral screens seemed easier thanks to the small overlap of corresponding landmarks on the endoscope and the ELViS screens. When asked, none of the participants indicated that the three-screen setup was a hindrance while performing the exercise. Some participants proposed an enhanced screen setup with a small side screen located to the right of the laparoscopic image, displaying the two images from the lateral cameras. This setup would add to the ergonomic advantages and convenience, as only one additional screen would be brought into the operating room. However, this type of display would considerably reduce the size of the images from the side cameras. 
The person manipulating the laparoscope (surgical assistant) must provide the appropriate point of view for the surgeon and display the surgical scene when required during surgery. One of the roles of the assistant is to align the endoscope by rotating the laparoscope to focus the narrow field of view correctly with respect to the background scene, and misalignments could adversely affect performance, especially for junior surgeons[28]. The ELViS could indirectly enhance learning of laparoscopic surgery by helping the surgical assistant. When asked, most surgeons felt that ELViS could represent a significant aid to the assistant. In our study, we saw that the ELViS did not affect how the assistant handled the endoscope in terms of total movement distance measured for this camera. In future experiments, emphasis will be placed on assessing the viewing axis.

\section{Conclusion}

The results presented in this paper describe the positive impact of providing additional visual information to surgeons for the early detection of an adverse event in a dry lab simulation, without affecting the time to complete a task. Use of the ELViS was considered to improve some aspects of the quality of surgery. Surgeons mentioned that they would prefer to use the ELViS for long and difficult surgery out of concern for the cost of the device. We plan to perform further studies to bring the ELViS into a clinical setting.

\section{Acknowledgments}

The authors would like to thank the participants: Dr Francois Istasse, Dr Mathieu Jolivet, Dr Edouard Girard, Dr Pierre Yves Sage, Pr Jean Alexandre Long, Dr Nicolas Taton, Dr Julio Abba, Dr Fabian Reche, Dr Fabien Stenard, Pr Mircea Chirica, Dr Camille Micoud, and Dr Sophie Gobillot. They would also like to thank the Centre d'Investigation Clinique - Innovation Technologique of Grenoble Alpes University Hospital and Maighread Gallagher-Gambarelli (TWS Editing) for Editorial support. 


\section{Disclosures}

Dr. Sandrine Voros is named as inventor on a patent EP2903495B1 (FR2996437B1) relating to the

ELViS. Dr Bertrand Trilling, Dr Sinara Vijayan, Dr Cecile Goupil, Elio Kedisseh, Dr Antoine Letouzey,

Pr Jean Luc Faucheron, and Dr Gaëlle Fiard have no conflicts of interest to disclose.

\section{References}

[1] Keus F, de Jong J, Gooszen HG, Laarhoven CJ. Laparoscopic versus open cholecystectomy for patients with symptomatic cholecystolithiasis. In: The Cochrane Collaboration, editor. Cochrane Database of Systematic Reviews, Chichester, UK: John Wiley \& Sons, Ltd; 2006.

[2] Beck TL, Schiff MA, Goff BA, Urban RR. Robotic, Laparoscopic, or Open Hysterectomy: Surgical Outcomes by Approach in Endometrial Cancer. Journal of Minimally Invasive Gynecology 2018;25:986-93. https://doi.org/10.1016/j.jmig.2018.01.010.

[3] Ratti F, Fiorentini G, Cipriani F, Catena M, Paganelli M, Aldrighetti L. Laparoscopic vs Open Surgery for Colorectal Liver Metastases. JAMA Surgery 2018;153:1028. https://doi.org/10.1001/jamasurg.2018.2107.

[4] Bonjer HJ, Deijen CL, Abis GA, Cuesta MA, van der Pas MHGM, de Lange-de Klerk ESM, et al. A Randomized Trial of Laparoscopic versus Open Surgery for Rectal Cancer. New England Journal of Medicine 2015;372:1324-32. https://doi.org/10.1056/NEJMoa1414882.

[5] van der Voort M, Heijnsdijk EAM, Gouma DJ. Bowel injury as a complication of laparoscopy. British Journal of Surgery 2004;91:1253-8. https://doi.org/10.1002/bjs.4716.

[6] Kaushik R. Bleeding complications in laparoscopic cholecystectomy: Incidence, mechanisms, prevention and management. Journal of Minimal Access Surgery 2010;6:59. https://doi.org/10.4103/0972-9941.68579.

[7] Nakano S, Nakahira J, Sawai T, Kadono N, Minami T. Unexpected hemorrhage during robotassisted laparoscopic prostatectomy: a case report. Journal of Medical Case Reports 2016;10. https://doi.org/10.1186/s13256-016-1030-4.

[8] Law KE, Lowndes BR, Kelley SR, Blocker RC, Larson DW, Hallbeck MS, et al. NASA-Task Load Index Differentiates Surgical Approach: Opportunities for Improvement in Colon and Rectal Surgery. Annals of Surgery 2018;271:906-12. https://doi.org/10.1097/SLA.0000000000003173.

[9] Garcia-Martinez A, Vicente-Samper JM, Sabater-Navarro JM. Automatic detection of surgical haemorrhage using computer vision. Artificial Intelligence in Medicine 2017;78:55-60. https://doi.org/10.1016/j.artmed.2017.06.002.

[10] Roulet P, Konen P, Villegas M, Thibault S, Garneau PY. 360 endoscopy using panomorph lens technology. In: Tearney GJ, Wang TD, editors., San Francisco, California: 2010, p. 75580T. https://doi.org/10.1117/12.842417.

[11] Kobayashi E, Masamune K, Sakuma I, Dohi T. A Wide-Angle View Endoscope System Using Wedge Prisms. In: Delp SL, DiGoia AM, Jaramaz B, editors. Medical Image Computing and Computer-Assisted Intervention - MICCAI 2000, vol. 1935, Berlin, Heidelberg: Springer Berlin Heidelberg; 2000, p. 661-8. https://doi.org/10.1007/978-3-540-40899-4_68.

[12] Tseng Y-C, Han P, Hsu H-C, Tsai C-M. A Flexible FOV Capsule Endoscope Design Based on Compound Lens. Journal of Display Technology 2016:1-1. https://doi.org/10.1109/JDT.2016.2615681.

[13] Naya Y, Nakamura K, Araki K, Kawamura K, Kamijima S, Imamoto T, et al. Usefulness of panoramic views for novice surgeons doing retroperitoneal laparoscopic nephrectomy: Panoramic views for novice surgeons. International Journal of Urology 2009;16:177-80. https://doi.org/10.1111/j.1442-2042.2008.02215.x. 
[14] Tamadazte B, Fiard G, Long J-A, Cinquin P, Voros S. Enhanced vision system for laparoscopic surgery. Conf Proc IEEE Eng Med Biol Soc 2013;2013:5702-5. https://doi.org/10.1109/EMBC.2013.6610845.

[15] Cao A, Darin Ellis R, Klein ED, Auner GW, Klein MD, Pandya AK. Comparison of a supplemental wide field of view versus a single field of view with zoom on performance in minimally invasive surgery. Surgical Endoscopy 2008;22:1445-51. https://doi.org/10.1007/s00464-0079627-8.

[16] Sumi Y, Egi H, Hattori M, Suzuki T, Tokunaga M, Adachi T, et al. A prospective study of the safety and usefulness of a new miniature wide-angle camera: the "BirdView camera system." Surgical Endoscopy 2019;33:199-205. https://doi.org/10.1007/s00464-018-6293-y.

[17] Arain NA, Cadeddu JA, Best SL, Roshek T, Chang V, Hogg DC, et al. A randomized comparison of laparoscopic, magnetically anchored, and flexible endoscopic cameras in performance and workload between laparoscopic and single-incision surgery. Surgical Endoscopy 2012;26:1170 80. https://doi.org/10.1007/s00464-011-2013-6.

[18] Kim J-J, Watras A, Liu H, Zeng Z, Greenberg J, Heise C, et al. Large-Field-of-View Visualization Utilizing Multiple Miniaturized Cameras for Laparoscopic Surgery. Micromachines 2018;9:431. https://doi.org/10.3390/mi9090431.

[19] Tamadazte B, Agustinos A, Cinquin P, Fiard G, Voros S. Multi-view vision system for laparoscopy surgery. International Journal of Computer Assisted Radiology and Surgery 2015;10:195203. https://doi.org/10.1007/s11548-014-1064-2.

[20] Xiao J, Cui Z, Fu M, Kong X, Tang L, Wang Z, et al. An ex vivo liver training model continuously perfused to simulate bleeding for suture skills involved in laparoscopic liver resection: development and validity. Surgical Endoscopy 2016;30:4553-61. https://doi.org/10.1007/s00464016-4792-2.

[21] Lee JY, Mucksavage P, Canales C, McDougall EM, Lin S. High Fidelity Simulation Based Team Training in Urology: A Preliminary Interdisciplinary Study of Technical and Nontechnical Skills in Laparoscopic Complications Management. Journal of Urology 2012;187:1385-91. https://doi.org/10.1016/j.juro.2011.11.106.

[22] Farnebäck G. Two-Frame Motion Estimation Based on Polynomial Expansion. In: Bigun J, Gustavsson T, editors. Image Analysis, vol. 2749, Berlin, Heidelberg: Springer Berlin Heidelberg; 2003, p. 363-70. https://doi.org/10.1007/3-540-45103-X_50.

[23] Bradski G. \{The OpenCV Library\}. Dr Dobb’s Journal of Software Tools 2000.

[24] on behalf of the EAES committees, Francis NK, Curtis NJ, Conti JA, Foster JD, Bonjer HJ, et al. EAES classification of intraoperative adverse events in laparoscopic surgery. Surgical Endoscopy 2018;32:3822-9. https://doi.org/10.1007/s00464-018-6108-1.

[25] Bishoff JT, Allaf ME, Kirkels W, Moore RG, Kavoussi LR, Schroder F. Laparoscopic Bowel Injury: incidence and clinical presentation. The Journal of Urology 1999;161:887-90. https://doi.org/10.1016/S0022-5347(01)61797-X.

[26] Picerno T, Sloan NL, Escobar P, Ramirez PT. Bowel injury in robotic gynecologic surgery: risk factors and management options. A systematic review. American Journal of Obstetrics and Gynecology 2017;216:10-26. https://doi.org/10.1016/j.ajog.2016.08.040.

[27] Rivas-Blanco I, Sánchez-de-Badajoz E, García-Morales I, Lage-Sánchez JM, Sánchez-Gallegos P, Pérez-del-Pulgar CJ, et al. Global vision system in laparoscopy. Actas Urológicas Españolas (English Edition) 2017;41:274-8. https://doi.org/10.1016/j.acuroe.2017.03.007.

[28] Rhee R, Fernandez G, Bush R, Seymour NE. The effects of viewing axis on laparoscopic performance: a comparison of non-expert and expert laparoscopic surgeons. Surgical Endoscopy 2014;28:2634-40. https://doi.org/10.1007/s00464-014-3515-9. 


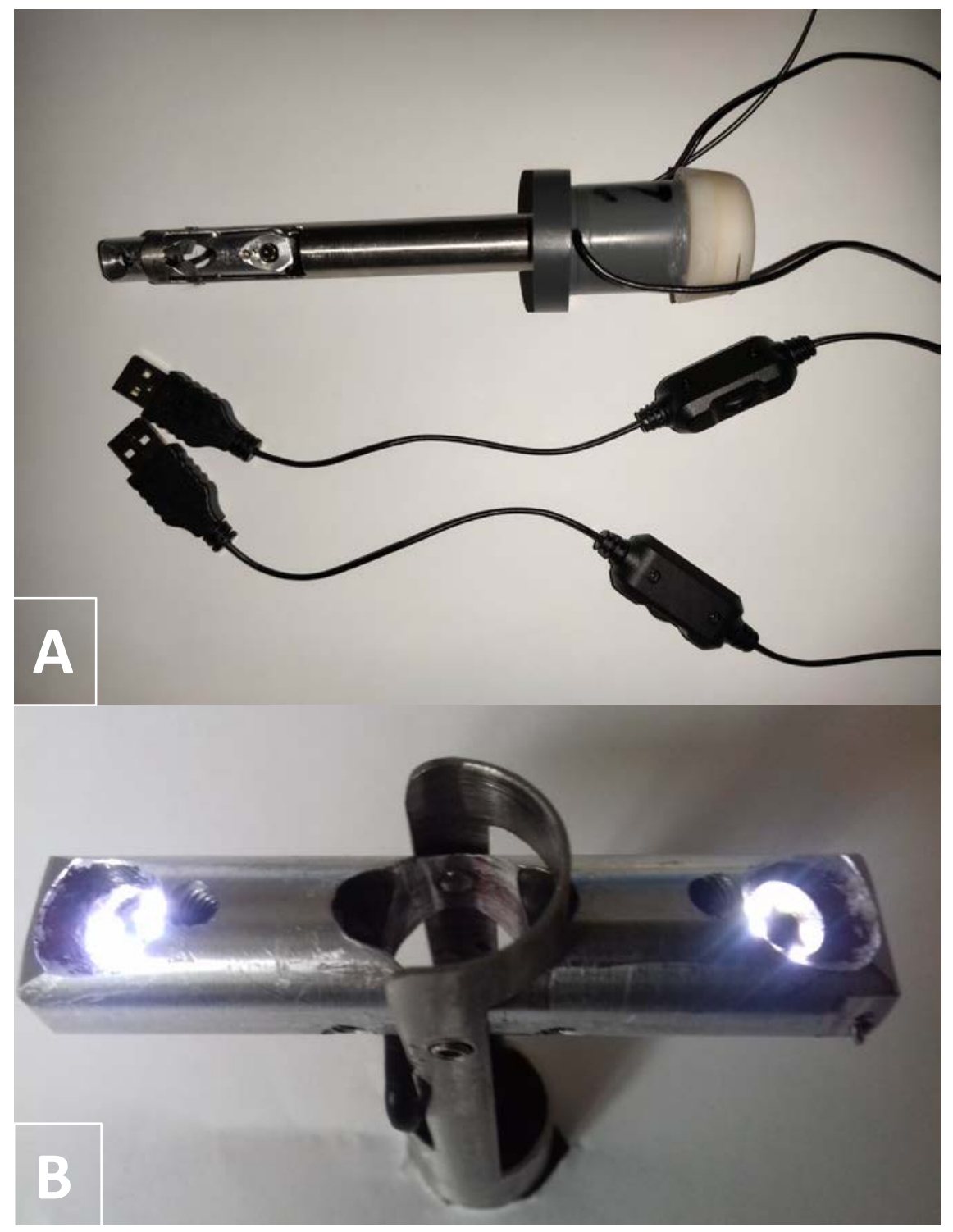

Figure 1. A. ELViS prototype in the folded position. The device is an enhanced trocar that is inserted through a 15-mm port and deployed inside the abdomen. The micro cameras with integrated LED placed in the camera module are connected to the computer via USB connectivity. B. ELViS prototype inserted through the upper plate of a laparoscopic simulator and deployed. The side cameras are placed as far as possible from the surgical scene to provide a wide view. 


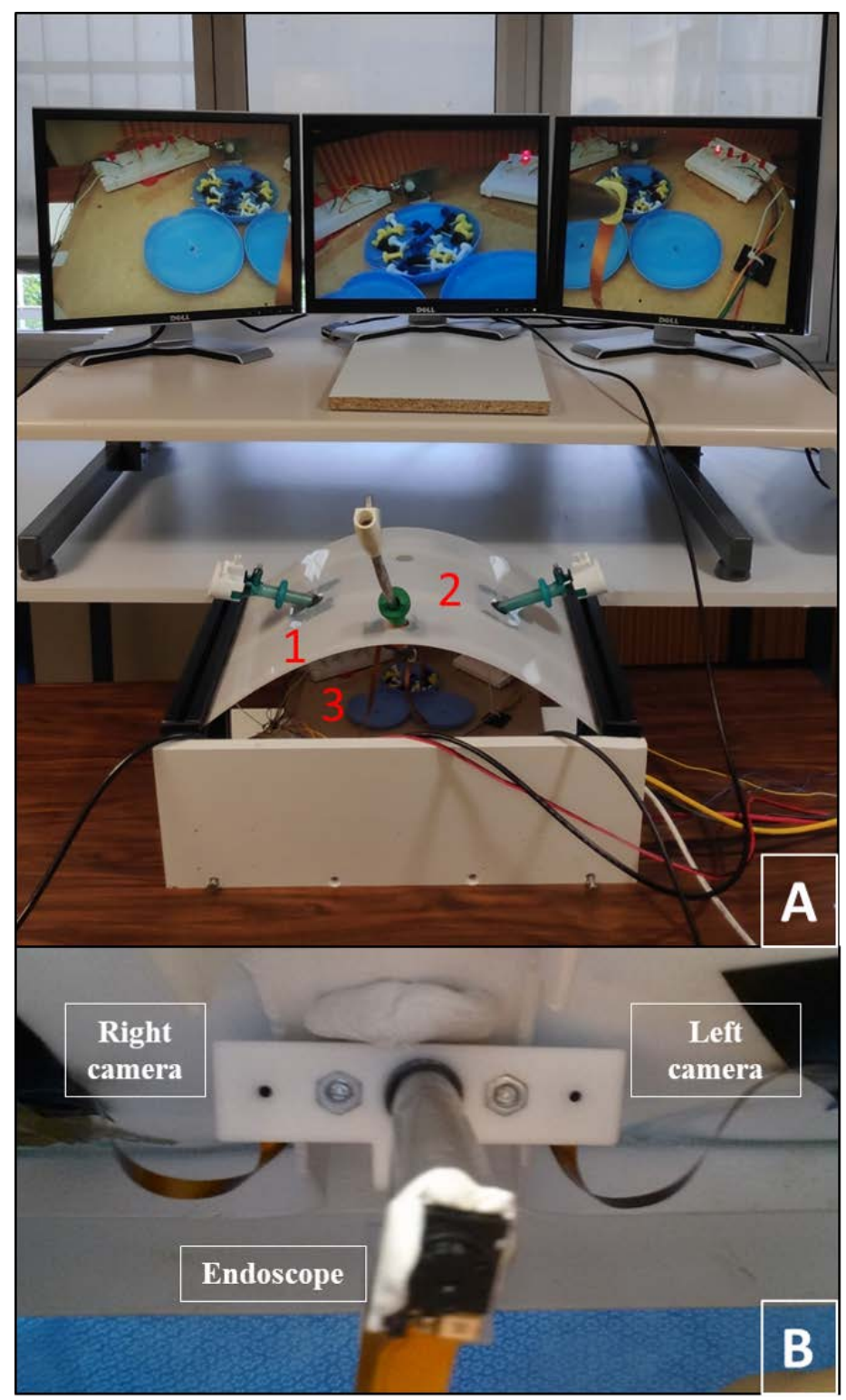

Figure 2. Dry lab setup with Enhanced Laparoscopic Vision. A. The three screens display the images from three cameras (left camera, classic endoscopic camera, right camera). Two intrument trocars (1) and one endoscope trocar (2) with the fixed, curved plastic plate mimicking the inflated abdomen designed for the task to be performed on the exercice plate (3). B. View from below of the plastic casing with the two mini-CMOS cameras, and the mock endoscope. 


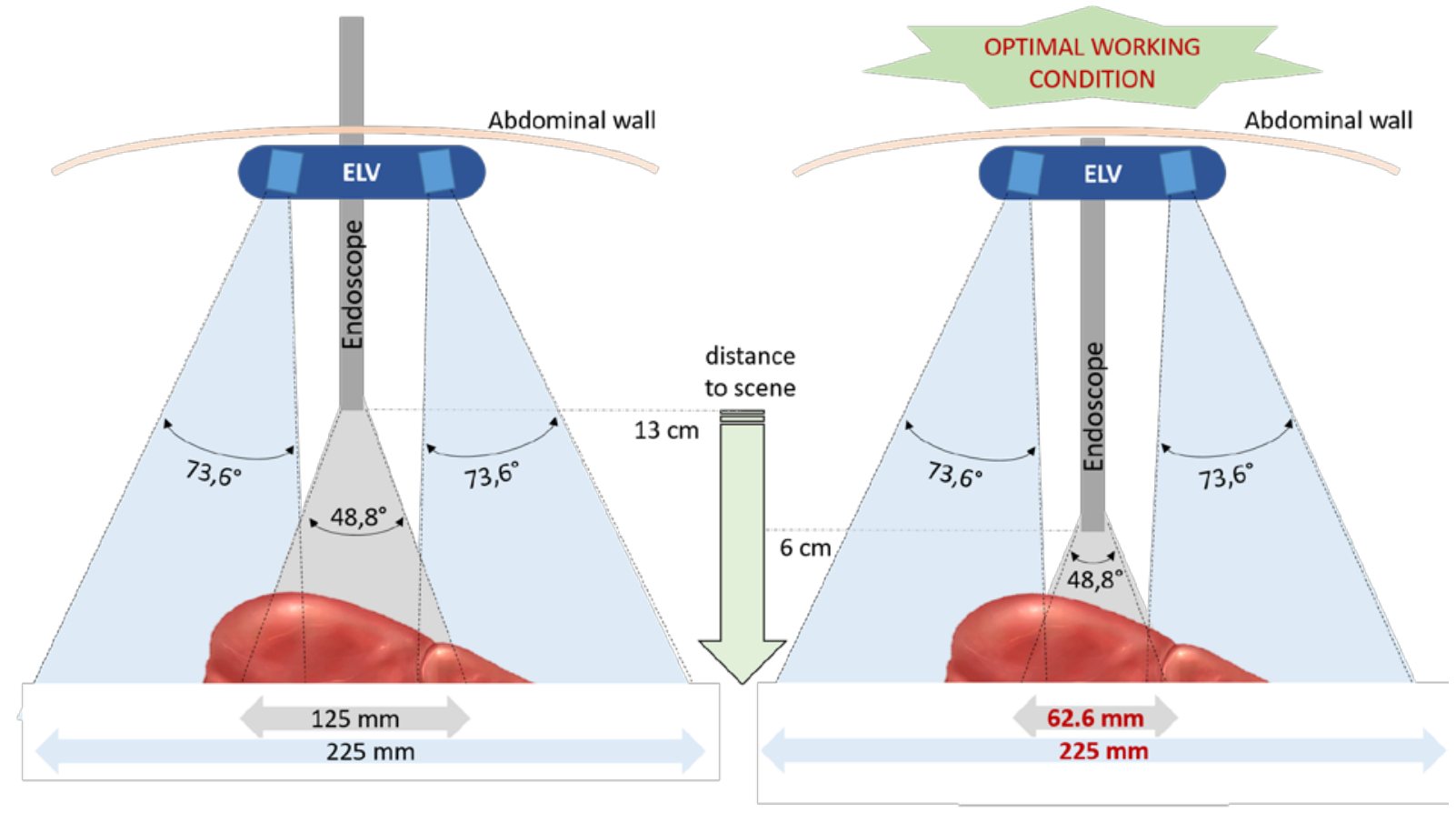

Figure 3. The endoscope can be moved vertically, and the tip of the endoscope can be approached or withdrawn from the surgical scene to zoom in or out. A. When distance between the tip of the endoscope and the surgical scene measures $13 \mathrm{~cm}$, the endoscope camera provides a $125-\mathrm{mm}$ horizontal field of view. B. When the distance between the tip of the endoscope and the surgical scene measures $6 \mathrm{~cm}$, the endoscope camera provides a $62.5-\mathrm{mm}$ horizontal field of view. The ELViS gives a $225-\mathrm{mm}$ horizontal field of view whatever the endoscope's position. 


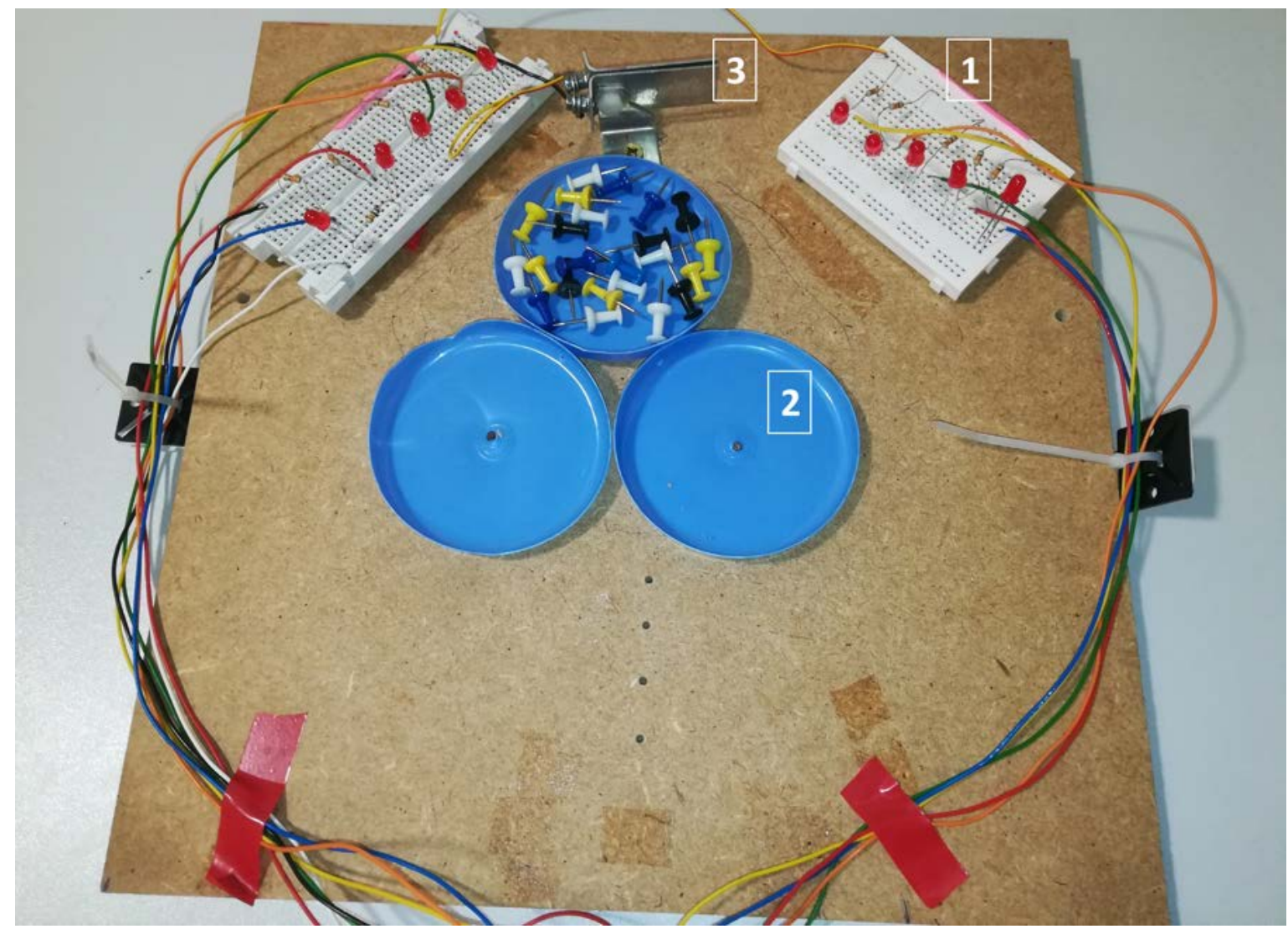

Figure 4. Exercise board designed to simulate bleeding during an intervention. 1) The two white breadboards (5 LEDs each) placed laterally. 2) The three central plates into which the 16 multi-colored pins were to be sorted. 3) The metal plate in the center between the two breadboards was the switch used to stop the simulated bleeding.

\begin{tabular}{|l|l|l|l|}
\hline & Endoscope only & ELV & p-value \\
\hline $\begin{array}{l}\text { Time to complete } \\
\text { exercise (in seconds) }\end{array}$ & $138.15(26.88)$ & $128.54(37.80)$ & 0.22 \\
\hline $\begin{array}{l}\text { Mean reaction time to } \\
\text { bleeding (in seconds) }\end{array}$ & $11.37(8.10)$ & $6.41(3.06)$ & 0.007 \\
\hline $\begin{array}{l}\text { Mean normalized } \\
\text { endoscope movement by } \\
\text { assistant (movement over } \\
\text { time) }\end{array}$ & $0.11(0.03)$ & $0.12(0.03)$ & 0.12 \\
\hline $\begin{array}{l}\text { Mean no. of } \\
\text { interruptions to control } \\
\text { simulated bleeding }\end{array}$ & 6.6 & 5.8 & 0.43 \\
\hline
\end{tabular}

Table 1. Time-to-completion, response time, number of interruptions, and mean normalized movement of the endoscope during simulated interventions. 


\begin{tabular}{|l|l|l|l|l|}
\hline & $\begin{array}{l}\text { Fully } \\
\text { agree }\end{array}$ & $\begin{array}{l}\text { Partially } \\
\text { agree }\end{array}$ & $\begin{array}{l}\text { Partially } \\
\text { disagree }\end{array}$ & $\begin{array}{l}\text { Fully } \\
\text { disagree }\end{array}$ \\
\hline The ELViS setup was not a hindrance during surgery & 10 & 3 & 0 & 0 \\
\hline The cognitive workload when using three screens seen is low & 6 & 5 & 0 \\
\hline $\begin{array}{l}\text { The conditions imposed during the exercise (display, } \\
\text { bleeding, avoiding organ damage) are similar to the } \\
\text { conditions encountered during typical laparoscopic surgery }\end{array}$ & 2 & 11 & 0 \\
\hline $\begin{array}{l}\text { This device would help to improve the quality of the surgical } \\
\text { procedure }\end{array}$ & 10 & 2 & 1 & 0 \\
\hline $\begin{array}{l}\text { The device helps manage bleeding during the procedure } \\
\text { The device helps handle obstacles better than during typical } \\
\text { laparoscopic surgery }\end{array}$ & 6 & 7 & 0 \\
\hline $\begin{array}{l}\text { The device helped the assistant to be more effective during } \\
\text { the experiment }\end{array}$ & 4 & 7 & 0 & 0 \\
\hline $\begin{array}{l}\text { This device will contribute to patient safety during surgery } \\
\text { The }\end{array}$ & 5 & 8 & 2 & 0 \\
\hline
\end{tabular}

Table 2. Summary of responses for each item of the questionnaire. 


\section{Supporting information captions:}

Appendix "Questionnaire": questionnaire submitted to the participating surgeons for the qualitative assessment of the Enhanced Laparoscopic Vision system

Video "ELV to improve laparoscopy": video showing the exercise performed for the evaluation, through the 3-screen interface. 